\title{
Effects of superstructure flexibility on the response of base-isolated structures
}

\author{
Jeevan A. Kulkarni and R.S. Jangid \\ Department of Civil Engineering, Indian Institute of Technology Bombay, Powai, Mumbai - 400 076, India
}

Received 7 June 2001

Revised 1 May 2002

\begin{abstract}
A parametric study of base-isolated structure with different isolation systems is conducted for investigating the effects of superstructure flexibility. The superstructure is idealized as a one-storey structure isolated by different systems such as elastomeric bearings (with and without lead core) and sliding systems. The governing equations of motion of the isolated structural system are derived and the response of the system is obtained for stochastic model of earthquake ground motion. The earthquake ground motion is modeled as a uniformly modulated non-stationary random process. The stochastic response of isolated structure is obtained using the state variable approach. An equivalent linearization technique is used for the approximate response of isolated structure with non-linear isolation systems. The mean square superstructure acceleration and bearing displacement of the system are plotted under different system parameters and compared with the corresponding response of rigid sup erstructure condition to study the influence of superstructure flexibility. The comparison of response is made under various isolation system parameters (i.e. isolation period, damping, yield strength of elastomeric bearings and friction coefficient of sliding systems). It is observed that the bearing displacement can accurately obtained by modelling the superstructure as a rigid body in a base-isolated structure. However, such approximation may under-estimates the superstructure acceleration under certain conditions.
\end{abstract}

Keywords: Base isolation, earthquake, elastomeric bearing, equivalent linearization, sliding system, non-stationary, superstructure flexibility

\section{Introduction}

Seismic isolation is an old design idea, proposing the decoupling of a structure or part of it, or even of equipment placed in the structure, from the damaging effects of ground accelerations. One of the goals of the seismic isolation is to shift the fundamental frequency of a structure away from the dominant frequencies of earthquake ground motion and fundamental frequency of the fixed base superstructure. The other purpose of an isolation system is to provide an additional means of energy dissipation, thereby reducing the transmitted acceleration into the superstructure. This innovative design approach aims mainly at the isolation of a structure from the supporting ground, generally in the horizontal direction, in order to reduce the transmission of the earthquake motion to the structure.

A variety of isolation devices including elastomeric bearings (with and without lead core), frictional/sliding bearings and roller bearings have been developed and used practically for aseismic design of buildings [1-3]. The laminated rubber bearings (LRB) base isolation system is the most common system consisting of alternate layers of rubber and steel plates [4]. To achieve high damping and initial rigidity a lead core is inserted in the LRB bearings known as the lead-rubber bearings which are widely used in New Zealand and are also referred as $\mathrm{N}-\mathrm{Z}$ system [5]. A significant amount of the recent research in base isolation has focused on the use of frictional elements to concentrate flexibility of the structural system and to add damping to the isolated structure. The most attractive feature of the frictional base isolation system is its effectiveness for a wide range of frequency input. The other advantage of a frictional type system is that it ensures the maximum acceleration transmissibility equal to the maximum limiting frictional force. The simplest sliding 
system device is pure-friction (P-F) system without any restoring force [6]. The sliding systems with restoring force include: the resilient-friction base isolator (R-FBI) system [7], Electricite de France (EDF) system [8], the friction pendulum system (FPS) [9], and the elliptical rolling rods [10]. Since the flexibility in the base-isolated structure is mainly concentrated in the isolation system, as a result, the response of baseisolated structure is investigated by modeling the superstructure as flexible [6,10-13] as well rigid [7,14,15]. The response of a base-isolated structure with rigid superstructure is quite simple (i.e., response analysis of a single degree-of-freedom (SDOF) system). However, it will be interesting to compare the response of a baseisolated structure with superstructure modeled as rigid and flexible to study the influence of superstructure flexibility.

Here-in, a comparison of the stochastic earthquake response of base-isolated structure with superstructure modelled as rigid and flexible is presented to study the effects of flexibility of the superstructure. The earthquake ground motion is considered to be non-stationary random process and the response of structure with different isolation systems is obtained using the state variable approach. In addition, a parametric study is also conducted to investigate the influence of important parameters on the difference of response of base- isolated structure with superstructure modelled as rigid and flexible.

\section{Model of base-isolated structure}

Consider a two mass structural model of a multistory building, as shown in Fig. 1(a) which had been widely considered in the past for the study of baseisolated structure $[6,13,16-18]$. The mass $m$ is intended to represent the superstructure of the building and $m_{b}$ the mass of the base floor above the isolation system. The building is assumed to have the linear forcedeformation behaviour with viscous damping. The structural stiffness and damping are represented by $k_{s}$, $c_{s}$ and the base mass by $m_{b}$. The superstructure and base mass can be completely be characterised by the three parameters, namely, $T_{s}, \xi_{s}$ and $\gamma$ defined as

$$
\begin{aligned}
& T_{s}=\frac{2 \pi}{\omega_{s}} \text { and } \omega_{s}=\sqrt{\frac{k_{s}}{m}} \\
& 2 \xi_{s} \omega_{s}=\frac{c_{s}}{m}
\end{aligned}
$$

$$
\gamma=\frac{m}{m+m_{b}}
$$

Consider the model of a base-isolated structure in which the superstructure is considered as a rigid body as shown in Fig. 1(b). In this model, the entire mass of the building is supported by the isolation system, as a result, it can be idealized as a SDOF system. A comparison of the response of two models will provide the influence of superstructure flexibility on the response of isolated structures. It is to be noted that the analysis of base-isolated structure with rigid superstructure has advantage in which a SDOF system is only to be analysed which is much simpler than the corresponding model with flexible superstructure.

\section{Model of earthquake excitation}

The earthquake excitation is considered as a uniformly modulated stationary Gaussian random process with zero-mean. The earthquake acceleration, $\ddot{x}_{g}(t)$ is expressed as

$$
\ddot{x}_{g}(t)=A(t) \ddot{u}_{f}(t)
$$

where $A(t)$ is the deterministic modulating function; and $\ddot{u}(t)$ is the stationary random process. The deterministic modulating function $A(t)$ of Eq. (4) is considered to be as shown in Fig. 2 from Reference [19]. This modulating function is expressed as

$$
A(t)= \begin{cases}\left(t / t_{1}\right)^{2} & \left(0 \leqslant t \leqslant t_{1}\right) \\ 1 & \left(t_{1} \leqslant t \leqslant t_{2}\right) \\ e^{-c\left(t-t_{2}\right)} & \left(t \geqslant t_{2}\right)\end{cases}
$$

where $t_{1}$ and $t_{2}$ denotes the time for start and end of strong motion duration, respectively; $c$ is a constant; and $T_{0}=t_{2}-t_{1}$ is known as strong motion duration of earthquake.

The evolutionary power spectral density function (PSDF) of the earthquake excitation is given as

$$
S_{\ddot{x}_{g}}(\omega)=|A(t)|^{2} S_{\ddot{u}_{f}}(\omega)
$$

where $\ddot{u}_{f}(\omega)$ is the stationary PSDF of the earthquake ground motion. In the present study, the PSDF of the earthquake excitation is considered as that suggested by Clough and Penzien [20] i.e.,

$$
\begin{aligned}
& S_{\ddot{u}_{f}}(\omega)=S_{0}\left(\frac{1+4 \xi_{g}^{2}\left(\omega / \omega_{g}\right)^{2}}{\left[1-\left(\omega / \omega_{g}\right)^{2}\right]^{2}+4 \xi_{g}^{2}\left(\omega / \omega_{g}\right)^{2}}\right) \\
& \left(\frac{\left(\omega / \omega_{f}\right)^{4}}{\left[1-\left(\omega / \omega_{f}\right)^{2}\right]^{2}+4 \xi_{f}^{2}\left(\omega / \omega_{f}\right)^{2}}\right)
\end{aligned}
$$




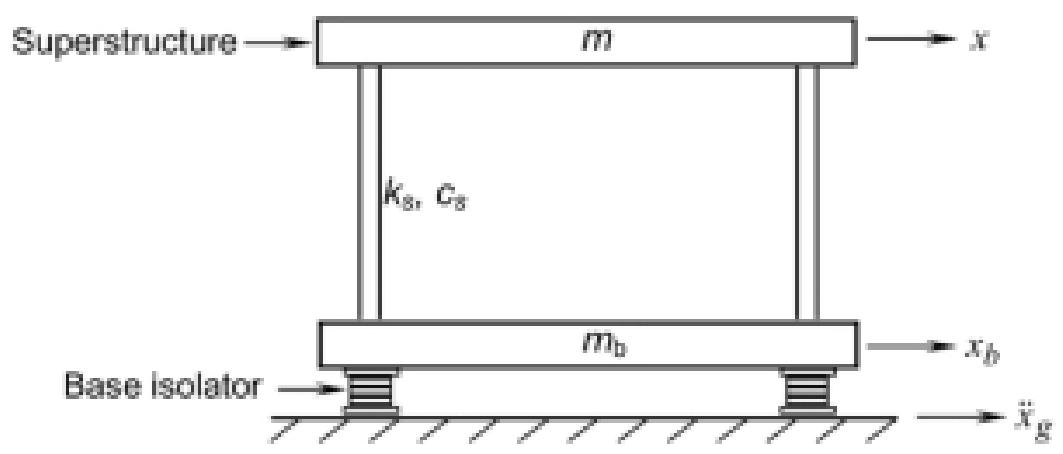

(a)

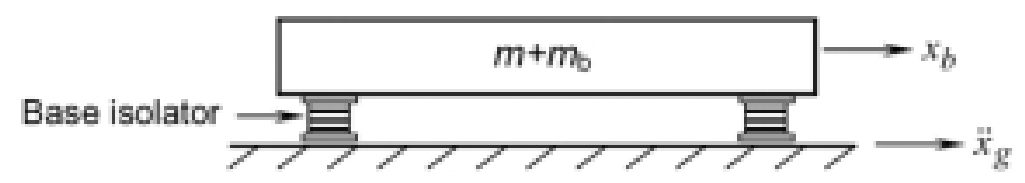

(b)

Fig. 1. Model of base-isolated structure with flexible and rigid superstructure.

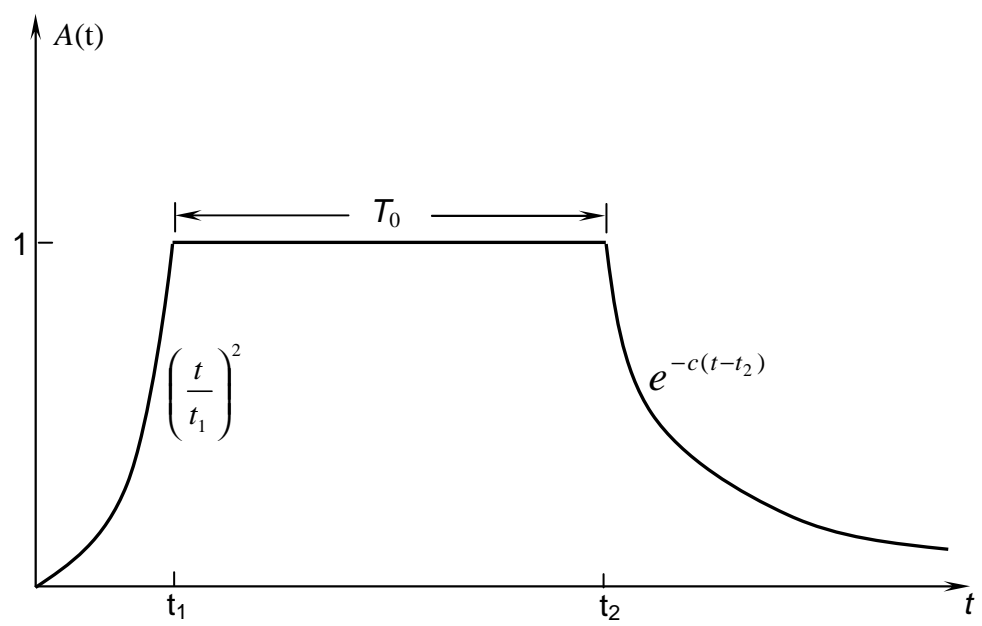

Fig. 2. Modulating function for non-stationary earthquake ground motion.

where $S_{0}$ is the constant PSDF of input white-noise random process; and $\omega_{g}, \xi_{g}, \omega_{f}$ and $\xi_{f}$ are the ground filter parameters.

The $\omega_{g}$ and $\xi_{g}$ generally represent the pre-dominant soil frequency and damping ratio. For the present study, the values of various parameters considered are: $\omega_{g}=$ $15.6 \mathrm{rad} / \mathrm{sec}, \xi_{g}=0.6, \omega_{f}=1.5 \mathrm{rad} / \mathrm{sec}, \xi_{f}=0.6$ and $S_{0}=0.05 \mathrm{~m}^{2} / \mathrm{sec}^{3}$ which represent earthquake ground motion in a firm soil [20]. The variation in the PSDF of with these parameters is shown in Fig. 3.

Note that the state variable method for stochastic re- sponse of any system requires that the excitation must be either white-noise or shot-noise whereas the PSDF of $\ddot{u}_{f}(t)$ is a non-white random process. However, this obstacle can be circumvented by introducing the shaping filters in which the random process, $\ddot{u}_{f}(t)$ can be considered as the response of two linear filters subjected to white-noise excitation as

$$
\begin{aligned}
& \ddot{u}_{f}(t)+2 \xi_{f} \omega_{f} \dot{u}_{f}(t)+\omega_{f}^{2} u_{f}(t)= \\
& \ddot{u}_{g}(t)+\ddot{u}_{0}(t) \\
& \ddot{u}_{g}(t)+2 \xi_{g} \omega_{g} \dot{u}_{g}(t)+\omega_{g}^{2} u_{g}(t)=-\ddot{u}_{0}(t)
\end{aligned}
$$




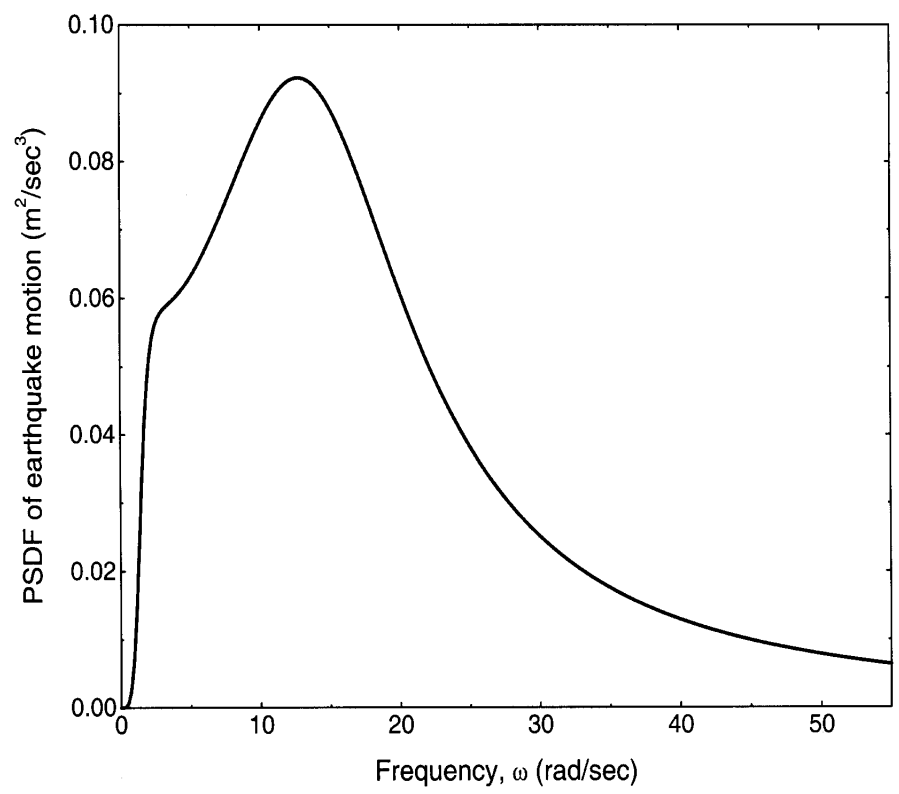

Fig. 3. Plot of PSDF of acceleration, $\ddot{u}_{f}(t)$ against the frequency $\left(\omega_{g}=15.6 \mathrm{rad} / \mathrm{sec}, \omega_{f}=1.5 \mathrm{rad} / \mathrm{sec}, \xi_{g}=0.6, \xi_{f}=0.6\right.$ and $\left.S_{0}=0.05 \mathrm{~m}^{2} / \mathrm{sec}^{3}\right)$.

where $\ddot{u}_{0}(t)$ is the input white-noise random process with constant intensity of the PSDF as $S_{0}$. Note that the Eqs. (8) and (9) provide the stationary PSDF of the response $\ddot{u}_{f}(t)$ as that expressed by the Eq. (7).

\section{Governign equations of motion}

The equation governing the motion of the superstructure mass to earthquake excitation is expressed as

$$
m \ddot{x}+c_{s}\left(\dot{x}-\dot{x}_{b}\right)+k_{s}\left(x-x_{b}\right)=-m \ddot{x}_{g}
$$

where $x$ is the displacement of the superstructure relative to ground; $x_{b}$ is the displacement of the base mass relative to the ground; and $\ddot{x}_{g}$ is the earthquake ground acceleration expressed by Eq. (4).

The equation of motion of the base mass is expressed by

$$
\begin{aligned}
& m_{b} \ddot{x}_{b}+F_{b}-c_{s}\left(\dot{x}-\dot{x}_{b}\right)-k_{s}\left(x-x_{b}\right)= \\
& -m_{b} \ddot{x}_{g}
\end{aligned}
$$

where $F_{b}$ is the restoring force of the isolation system.

The corresponding governing equation of motion of the isolated rigid superstructure as shown in Fig. 1(b) is expressed by

$$
\left(m+m_{b}\right) \ddot{x}_{b}+F_{b}=-\left(m+m_{b}\right) \ddot{x}_{g}
$$

Substituting for $c_{s}\left(\dot{x}-\dot{x}_{b}\right)+k_{s}\left(x-x_{b}\right)$ in Eq. (11) from Eq. (10), the Eq. (11) reduces to

$$
\left(m+m_{b}\right) \ddot{x}_{b}+F_{b}+m \ddot{x}_{r}=-\left(m+m_{b}\right) \ddot{x}_{g}
$$

where $\ddot{x}_{r}=\ddot{x}-\ddot{x}_{b}$ is the acceleration of the superstructure relative to the base mass.

By comparing the Eqs (12) and (13), there is a difference of the term $m \ddot{x}_{r}$ in the governing equation of motion of the base mass when the superstructure is idealized as rigid and flexible. Since the superstructure of the base-isolated buildings is relatively stiff in comparison to the isolation system, as a result, the relative displacement, $x_{r}$ approaches to zero which justifies the analysis of base-isolated structure with rigid superstructure.

The restoring force, $F_{b}$ depends upon the type of isolation system considered and for various types of isolation systems it is described below.

\subsection{Laminated rubber bearings}

The LRB system is the most common isolation system consisting of alternate layers of steel and rubber plates. The dominant features of LRB system are the parallel action of linear spring and damping. Generally, the LRB system exhibits high-damping capacity, horizontal flexibility and high vertical stiffness. The LRB system is modeled by linear force-deformation behaviour with viscous damping. The restoring force is expressed by

$$
F_{b}=c_{b} \dot{x}_{b}+k_{b} x_{b}
$$




$$
\begin{aligned}
& {[\mathrm{H}]=\left\{\begin{array}{llllllll}
0 & 1 & 0 & 0 & 0 & 0 & 0 & 0 \\
-\frac{k_{s}}{m}-\frac{c_{s}}{m} & \frac{k_{s}}{m} & \frac{c_{s}}{m} & -\omega_{f}^{2} A(t)-2 \xi_{f} \omega_{f} A(t)-\omega_{g}^{2} A(t)-2 \xi_{g} \omega_{g} A(t) \\
0 & 0 & 0 & 1 & 0 & 0 & 0 & 0 \\
\frac{k_{s}}{m_{b}} & \frac{c_{s}}{m_{b}} & -\frac{k_{s}+k_{b}}{m_{b}} & -\frac{c_{s}+c_{b}}{m_{b}} & -\omega_{f}^{2} A(t)-\xi_{f} \omega_{f} A(t) & -\omega_{g}^{2} A(t)-2 \xi_{g} \omega_{g} A(t) \\
0 & 0 & 0 & 0 & 0 & 1 & 0 & 0 \\
0 & 0 & 0 & 0 & -\omega_{f}^{2} & -2 \xi_{f} \omega_{f} & -\omega_{g}^{2} & -2 \xi_{g} \omega_{g} \\
0 & 0 & 0 & 0 & 0 & 0 & 0 & 1 \\
0 & 0 & 0 & 0 & 0 & 0 & -\omega_{g}^{2} & -2 \xi_{g} \omega_{g}
\end{array}\right]} \\
& {[\mathrm{H}]=\left\{\begin{array}{lllllll}
0 & 1 & 0 & 0 & 0 & 0 & \\
-\frac{k_{b}}{m+m_{b}}-\frac{c_{b}}{m+m_{b}}-\omega_{f}^{2} A(t)-2 \xi_{f} \omega_{f} A(t)-\omega_{g}^{2} A(t)-2 \xi_{g} \omega_{g} A(t) \\
0 & 0 & 0 & 1 & 0 & 0 \\
0 & 0 & -\omega_{f}^{2} & -2 \xi_{f} \omega_{f} & -\omega_{g}^{2} & -2 \xi_{g} \omega_{g} \\
0 & 0 & 0 & 0 & 0 & 1 \\
0 & 0 & 0 & 0 & -\omega_{g}^{2} & -2 \xi_{g} \omega_{g}
\end{array}\right]}
\end{aligned}
$$

Where $c_{b}$ and $k_{b}$ are the damping and stiffness of the LRB system, respectively.

The most convenient way to determine the response statistics of base-isolated structure is by using the state variable formulation. The Eqs (10) and (11) along with Eqs (4), (8) and (9) can be re-written as a system of first order stochastic differential equations as

$$
\frac{d}{d t}\{Y\}=[H]\{Y\}+\{F\}
$$

where

$$
\begin{aligned}
& \{Y\}=\left\{x, \dot{x}, x_{b}, \dot{x}_{b}, x_{f}, \dot{x}_{f}, x_{g}, \dot{x}_{g}\right\}^{T} \\
& \{F\}=\left\{0,0,0,0,0,0,0,-\ddot{u}_{0}\right\}^{T}
\end{aligned}
$$

The augmented response vector $\{Y\}$ is a Markov process and the corresponding covariance matrix $[V]$ satisfies [21] the following differential equation

$$
\frac{d}{d t}[V]=[H][V]+[V][H]^{T}+[P]
$$

where the elements of the matrix $[P]$ and $[V]$ are given as

$$
\begin{array}{r}
P_{i j}=E\left[F_{i} Y_{j}\right] \\
V_{i j}=E\left[Y_{i} Y_{j}\right]
\end{array}
$$

where $E$ is expectation operator.

The corresponding matrices $\{Y\},[H]$ and $\{F\}$ for a rigid superstructure isolated by the LRB system are expressed by

$$
\begin{aligned}
& \{Y\}=\left\{x_{b}, \dot{x}_{b}, x_{f}, \dot{x}_{f}, x_{g}, \dot{x}_{g}\right\}^{T} \\
& \{F\}=\left\{0,0,0,0,0,-\ddot{u}_{0}\right\}^{T}
\end{aligned}
$$

The elements of the matrix $[P]$ are $P_{i j}=0$ except the $P_{88}$ for flexible superstructure and $P_{66}$ for rigid superstructure which are equal to $2 \pi S_{0}$. The non-stationary response of the system (i.e., $[V]$ matrix) is obtained by solving the moment Eq. (19). The augmented system matrix, $[H]$ is time dependent through the introduction of the modulating function, $A(t)$. Therefore, the Eq. (19) is solved numerically based on step-by-step method using fourth order Runge-Kutta method.

\section{2. $N$-Z system}

The N-Z system provides additional hysteretic damping through the yielding of the lead core. The hysteresis loop of a bearing is generally modelled by bi-linear force-deformation behaviour expressed by the Wen's equation [22]. The restoring force is expressed by

$$
F_{b}=c_{b} \dot{x}_{b}+\alpha k_{b} x_{b}+(1-\alpha) F_{y} Z
$$

where $c_{b}$ is the viscous damping of the bearing or the damping provided by additional viscous dampers; $k_{b}$ is the initial stiffness of the bearing; $\alpha$ is an index, which represents the ratio of post to pre-yielding stiffness; $F_{y}$ is the yield strength of the bearing; and $Z$ is a non-dimensional hysteretic component satisfying the following non-linear first order differential equation, which is expressed [22] as

$$
q \dot{Z}=A \dot{x}_{b}+\beta\left|\dot{x}_{b}\right| Z|Z|^{n-1}-\tau \dot{x}_{b}|Z|^{n}
$$

where $q$ is the yield displacement of the bearing; $\beta, \tau$ and $A$ are non-dimensional parameters of the hysteresis loop. The parameters $\alpha, \beta, \tau, A$ and $n$ control the shape of the loop and are selected such that predicted response from the model closely matches with the experimental results. 
The Eq. (26) is a non-linear equation, which cannot be used when the response is to be evaluated using state variable approach. Thus, the Eq. (26) is replaced by equivalent linear equation [23] as

$$
q \dot{Z}+C_{e} \dot{x}_{b}+K_{e} Z=0
$$

where $C_{e}$ and $K_{e}$ are the equivalent constants which are obtained by minimizing the mean square error between the linear and non-linear terms. For $n=1$, the equivalent constants $C_{e}$ and $K_{e}$ are given by

$$
\begin{aligned}
& C_{e}=\sqrt{\frac{2}{\pi}}\left\{\gamma \frac{E\left(\dot{x}_{b}, Z\right)}{\sqrt{E(Z, Z)}}+\right. \\
& \left.\beta \sqrt{E\left(\dot{x}_{b}, \dot{x}_{b}\right)}\right\}-A \\
& K_{e}=\sqrt{\frac{2}{\pi}}\left\{\gamma \sqrt{E(Z, Z)}+\beta \frac{E\left(\dot{x}_{b}, Z\right)}{\sqrt{E(Z, Z)}}\right\}
\end{aligned}
$$

The stochastic response of the system can be obtained by the state variable method by solving the corresponding moment equation similar to Eq. (19). The corresponding state vector, $\{Y\}$ will be $\left\{x, \dot{x}, x_{b}, \dot{x}_{b}, Z, x_{f}, \dot{x}_{f}, \dot{x}_{f}, x_{g}, \dot{x}_{g}\right\}^{T}$ (for flexible superstructure) and $\left\{x_{b}, \dot{x}_{b}, Z, x_{f}, \dot{x}_{f}, x_{g}, \dot{x}_{g}\right\}^{T}$ (for rigid superstructre). It is to be noted that the non-linear phenomenon of the N-Z system still exists due to dependence of equivalent constant $C_{e}$ and $K_{e}$ on the elements of the $[V]$ matrix of the system (refer Eqs (28) and (29)). However, this is taken care by modifying the $C_{e}$ and $K_{e}$ in each time step depending upon the response of the system.

\subsection{Sliding Systems}

The restoring force for the sliding system is expressed by

$$
F_{b}=c_{b} \dot{x}_{b}+k_{b} x_{b}+F_{x}
$$

where $c_{b}$ and $k_{b}$ are the damping and stiffness of the sliding system; and $F_{x}$ is the frictional force in the sliding system.

If it is assumed that the system remains most of the time in the sliding phase than the friction force can be expressed as

$$
F_{x}=\mu\left(m_{b}+m\right) g \operatorname{sgn}\left(\dot{x}_{b}\right)
$$

where $\mu$ is coefficient of friction of the sliding system which is assumed to be independent of the relative sliding velocity.
Note that the $F_{x}$ is non-linear and is replaced by the equivalent linear term as

$$
F_{x}=c_{e} \dot{x}_{b}
$$

and

$$
c_{e}=\sqrt{\frac{2}{\pi}} \frac{\mu\left(m_{b}+m\right) g}{\sigma_{\dot{x}_{b}}}
$$

where $\sigma_{\dot{x}_{b}}$ is the root mean square (RMS) velocity of the base mass.

The stochastic response of the isolated structure with sliding system can be obtained by the similar approach as described for the LRB and N-Z systems.

\section{System parameters}

The superstructure of the isolated structural system requires the specification of two parameters namely, the fundamental time period of the superstructure with fixed base $\left(T_{s}\right)$ and the damping ratio of the superstructure $\left(\xi_{s}\right)$. The base mass can be designed by the specification of the parameter mass ratio $(\gamma)$. For the present study, the superstructure damping and mass ratio are held constant as these parameters does not have significant effects on the response of the isolated structure. The values taken are $\xi_{s}=0.02$ and $\gamma=0.5$ which are widely used in the past $[6,11,17]$. The fundamental time period of the structure, $T_{s}$ is varied between 0.1 to $1 \mathrm{sec}$ (typical fundamental time period of 1 to 10 storey building) for which the base isolation is effective.

The isolator parameters for the structure isolated by the LRB system requires the specification of the parameters such as the period of base isolation $\left(T_{b}\right)$ and the damping ratio of the bearing $\left(\xi_{b}\right)$ which are defined as between Eqs (34) and (35).

$$
\begin{aligned}
& T_{b}=\frac{2 \pi}{\omega_{b}} \text { and } \omega_{b}=\sqrt{\frac{k_{b}}{m+m_{b}}} \\
& 2 \xi_{b} \omega_{b}=\frac{c_{b}}{m+m_{b}}
\end{aligned}
$$

The N-Z system is generally designed to specified values of three parameters namely: the isolation period, $T_{b}$, the damping ratio, $\xi_{b}$ and the normalized yield strength, $F_{0}$. The parameters $T_{b}$ and $\xi_{b}$ are obtained from Eqs (34) and (35), respectively based on the postyield stiffness of the $\mathrm{N}-\mathrm{Z}$ system. The parameter, $F_{0}$ is defined as

$$
F_{0}=\frac{F_{y}}{\left(m+m_{b}\right) g}
$$



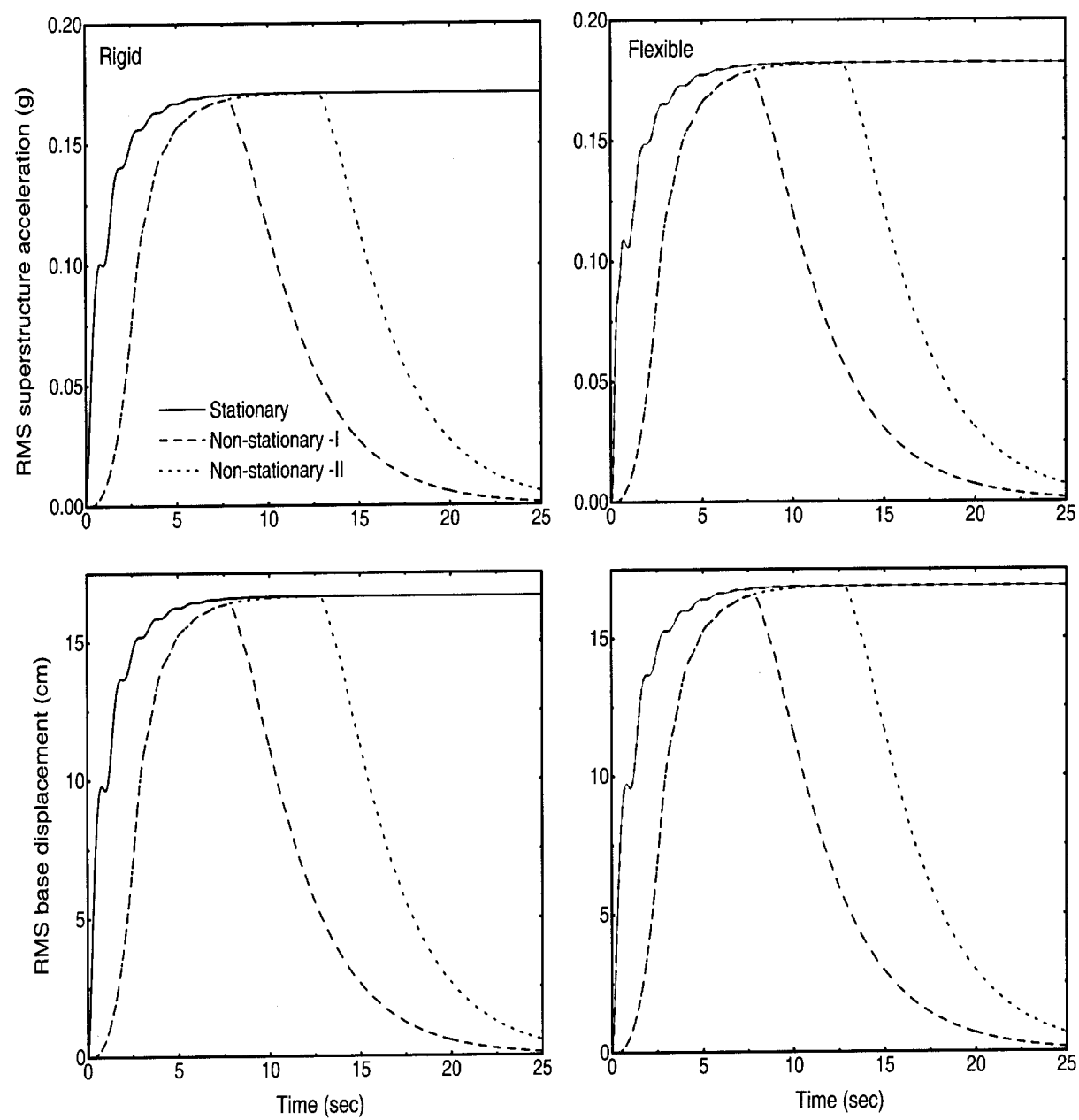

Fig. 4. Time variation of the RMS superstructure acceleration and base displacement of the structure isolated by the LRB system $(T=0.5$ sec, $T_{b}=2 \mathrm{sec}$ and $\left.\xi_{b}=0.1\right)$.

The other parameters of the N-Z system are the yield displacement level of the bearing $(q)$, and the parameters of hysteresis loop of the bearing such as $\beta, \tau, A$ and $n$. However, these parameters are held constant and values taken are: $q=25 \mathrm{~mm}, \beta=\tau=0.5, A=1$ and $n=1$ which are typical values used in the past.

The stiffness and viscous damping of the sliding system are evaluated to provide the required values of the parameters isolation period, $T_{b}$, damping ratio, $\xi_{b}$ (refer Eqs (34) and (35)). Thus, the sliding system requires the specifications of the three parameters namely the $T_{b}, \xi_{b}$ and $\mu$.

\section{Numerical study}

The response of both flexible and rigid superstructure isolated by different isolation systems to non-stationary earthquake motion is investigated. The non-stationary response of the system is obtained by solving Eq. (19) for different isolation systems and superstructure conditions. The PSDF function of the ground motion is considered as shown in Fig. 3. However, the effect of $\omega_{g}$ (i.e. predominant soil frequency) on the response of structure is studied separately. The response of the system is investigated for two types of modulating functions (as given in Fig. 2 and expressed by Eq. (5)) namely (i) $t_{1}=2.5 \mathrm{sec}, t_{2}=7.5 \mathrm{sec}$ and $c=0.5 \mathrm{sec}^{-1}$ and (iii) $t_{1}=2.5 \mathrm{sec}, t_{2}=12.5 \mathrm{sec}$ and $c=0.5 \mathrm{sec}^{-1}$. These modulating functions are referred as type $-\mathrm{I}$ and - II which has the strong motion duration, $T_{0}$ as 5 and $10 \mathrm{sec}$, respectively. In addition, the response is also investigated for $A(t)=1$ for all values of $t$ (this corresponds to a stationary earthquake ground motion) in order to distinguish between the stationary and non- 

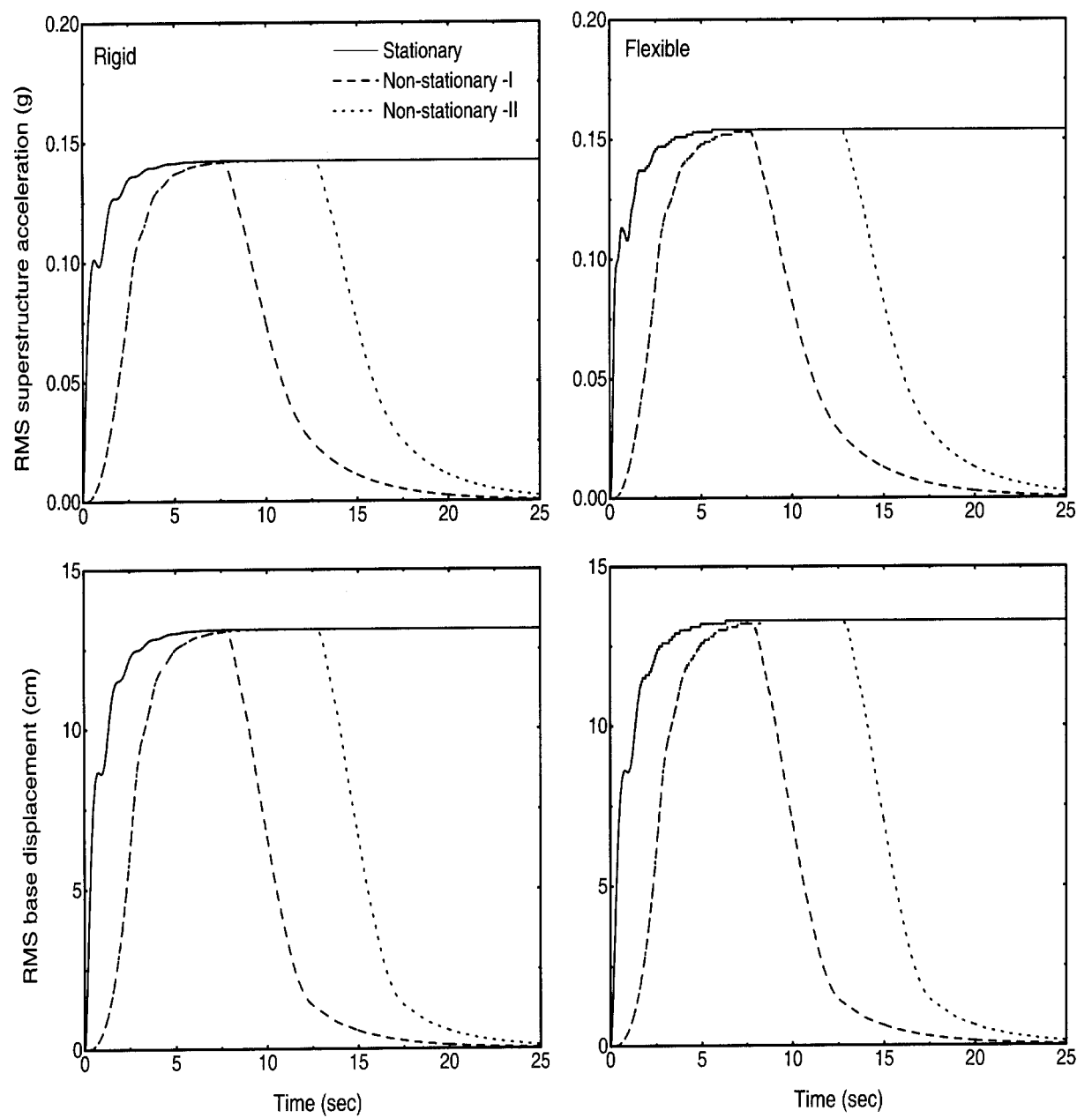

Fig. 5. Time variation of the RMS superstructure acceleration and base displacement of the structure isolated by the $\mathrm{N}-\mathrm{Z}$ system $\left(T_{8}=0.5\right.$ sec, $T_{b}=2.5 \mathrm{sec}, \xi_{b}=0.1$ and $\left.F_{0}=0.05\right)$.

stationary response of the base-isolated structures. For the base-isolated structure the response quantities of interest are the RMS absolute acceleration of the superstructure and the relative base displacement. The absolute acceleration is directly proportional to the forces exerted in the superstructure due to earthquake ground motion. On the other hand, the relative base displacement is crucial from the design point of view of the isolation system.

In Fig. 4, time variation of the RMS superstructure acceleration and base displacement of the structure isolated by the LRB system with $T_{b}=2 \mathrm{sec}$ and $\xi_{b}=0.1$ is plotted. The response is shown for considering the superstructure to be rigid as well as flexible (i.e. $T_{s}=0.5 \mathrm{sec}, \xi_{s}=0.02$ and $\gamma=0.5$ ) for different types of modulating functions. It is observed from the figure that the stationary response is achieved in a very short time (i.e. about $5 \mathrm{sec}$ ). In addition, the peak RMS response under non-stationary ground motion is the same as that of the stationary response. This happens mainly due to large damping in the base-isolated structure. Thus, the stochastic earthquake response of the base-isolated structures can be obtained by considering the stationary model of earthquake ground motion with appropriate frequency variation of PSDF function and intensity.

Further, the Fig. 4 also indicates that the RMS base displacement obtained under the rigid and flexible superstructure condition are almost identical implying that the rigid superstructure condition can be accurately used for predicting the bearing displacement. However, the RMS superstructure acceleration obtained with flexible superstructure is relatively higher than that obtained considering the rigid superstructure conditions. 

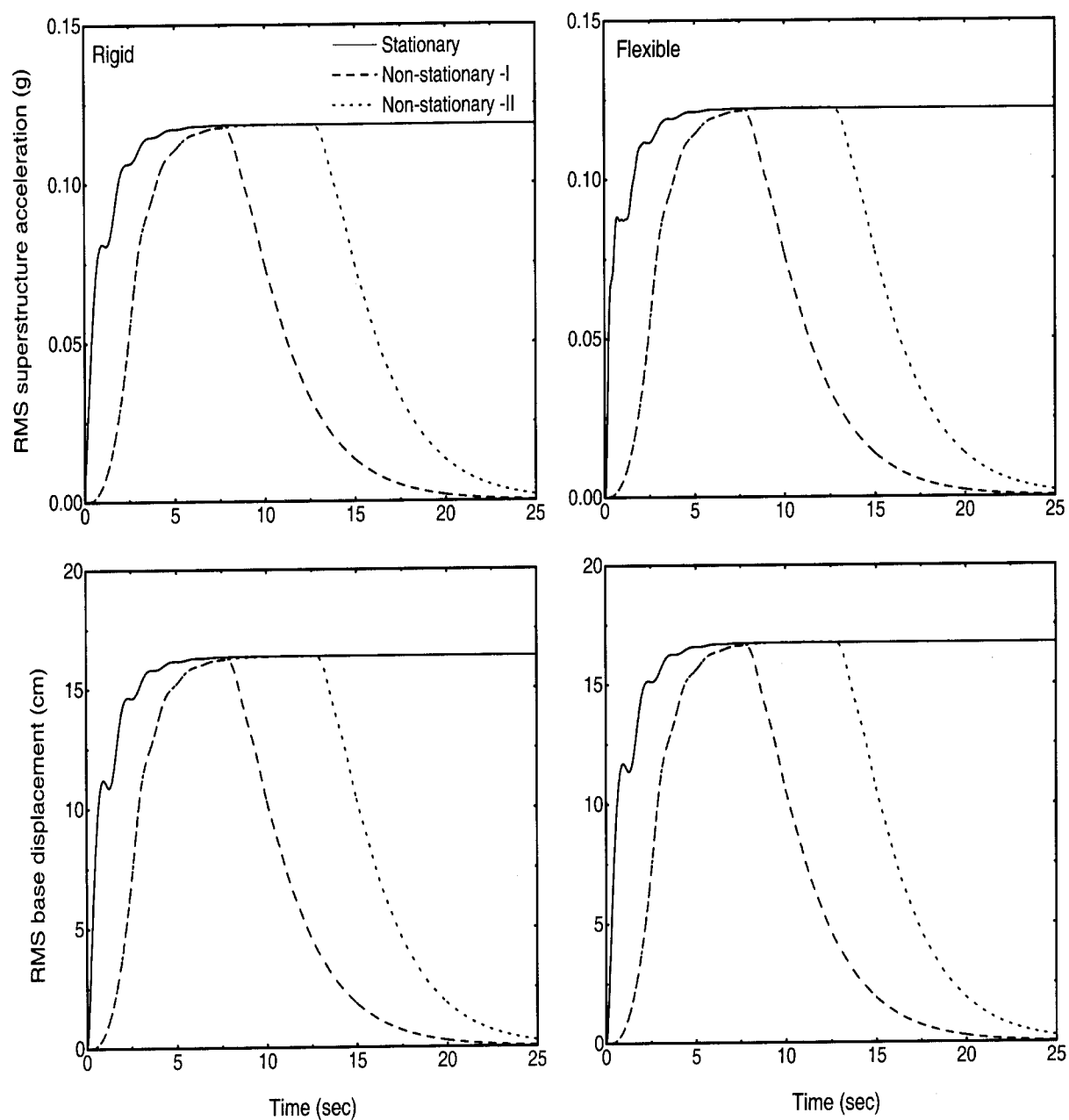

Fig. 6. Time variation of the RMS superstructure acceleration and base displacement of the structure isolated by the FPS system $\left(T_{8}=0.5\right.$ sec, $T_{b}=2.5 \mathrm{sec}$ and $\left.\mu=0.05\right)$.

This is due to the fact that in flexible superstructure there is a contribution from the second mode of vibration towards the absolute acceleration of superstructure but this mode has little contribution towards the base displacement [16]. Thus, the superstructure acceleration may be under-estimated if the flexibility of superstructure is ignored and it is modelled as a rigid body. Similar effects of the flexibility of superstructure and modulating functions are depicted in Figs 5 and 6 for the response of the structure isolated by the N-Z and FPS systems, respectively. The parameters considered for the N-Z system are $T_{b}=2.5 \mathrm{sec}, \xi_{b}=0.1$ and $F_{0}=0.05$ and for the FPS system the parameters taken are $T_{b}=2.5 \mathrm{sec}$ and $\mu=0.05$.

Figure 7 shows the variation of the peak RMS response of the structure isolated by the LRB system against the time period of the superstructure, $T_{s}$ for dif- ferent values of isolation period (i.e. $T_{b}=2,2.5$ and 3 sec) and bearing damping ratios (i.e. $\xi_{b}=0.1$ and 0.3 ). The response for $T_{s}=0$ is also shown which represent the corresponding response of the rigid superstructure condition. The figure indicates that the RMS superstructure acceleration of the structure remains constant for increase of the superstructure time period upto a certain value (i.e. about 0.4 to $0.5 \mathrm{sec}$ ). However, increase in the time period beyond this value will increase the superstructure acceleration. Thus, the superstructure acceleration can be estimated accurately using the rigid superstructure approach if the superstructure time period is one-fifth of the isolation period. Further, the difference between the superstructure acceleration obtained by modelling the superstructure as flexible and rigid increa ses with the increase of the bearing damping. The RMS base displacement of the base-isolated 

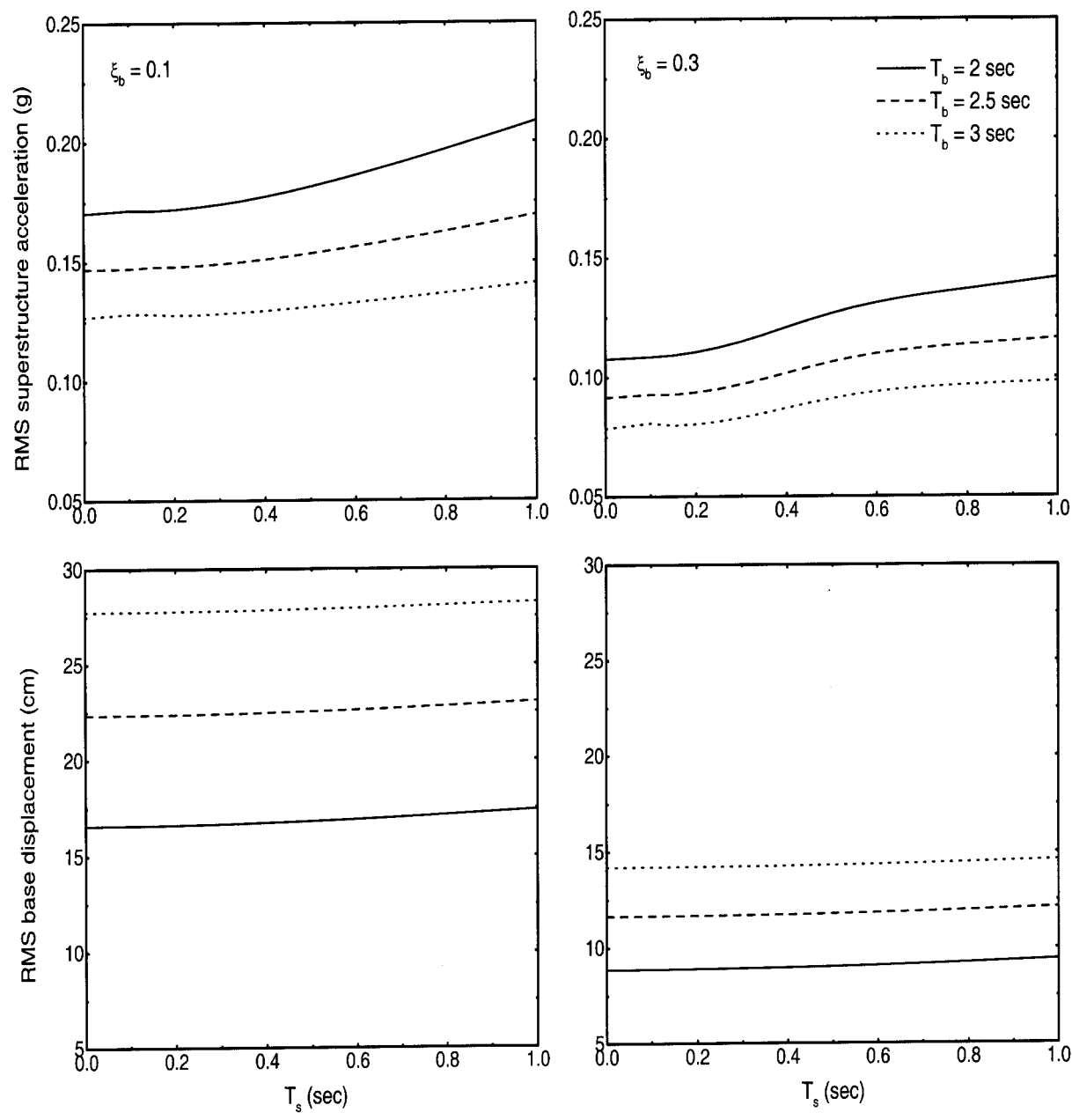

Fig. 7. Effects of the superstructure flexibility on the peak response of structure isolated by the LRB system.

structure remains invariant for different time periods of the superstructure. This implies that the rigid superstructure approach can effectively used for evaluating the RMS base displacement of a base-isolated structure.

The variation of peak RMS superstructure acceleration and base displacement against the superstructure time period is shown in Figs 8 and 9 for the structure isolated by the N-Z and FPS system, respectively. The effects of the superstructure time period on the peak RMS response are similar to that observed in the Fig. 7 for the LRB system. However, the difference between the superstructure acceleration obtained by modelling the superstructure as flexible and rigid increases with the increase of the yield strength level of the N-Z system and coefficient of friction of the FPS system. This is expected because of the increase of these parameters the isolation system becomes relatively stiffer, as a result, the contribution to superstructure acceleration from its own flexibility increases.

So far the response of the isolated structure is obtained for fixed value of the pre-dominant soil frequency, $\omega_{g}$ and it will be interesting to study the effects of $\omega_{g}$ on the difference between the response of isolated structure with superstructure modelled as rigid and flexible. In Fig. 10, the peak RMS superstructure acceleration and the base displacement of the structure isolated by different isolation systems are plotted against the soil frequency. It is observed from the figure that the RMS superstructure acceleration as well as base displacement decreases with the increase of $\omega_{g}$ implying that the seismic isolation is more effective for firm or rock type soil than the soft soils. Further, the difference in the response between flexible and rigid superstructure modelling is not much influenced by the pre-dominant ground frequency. 

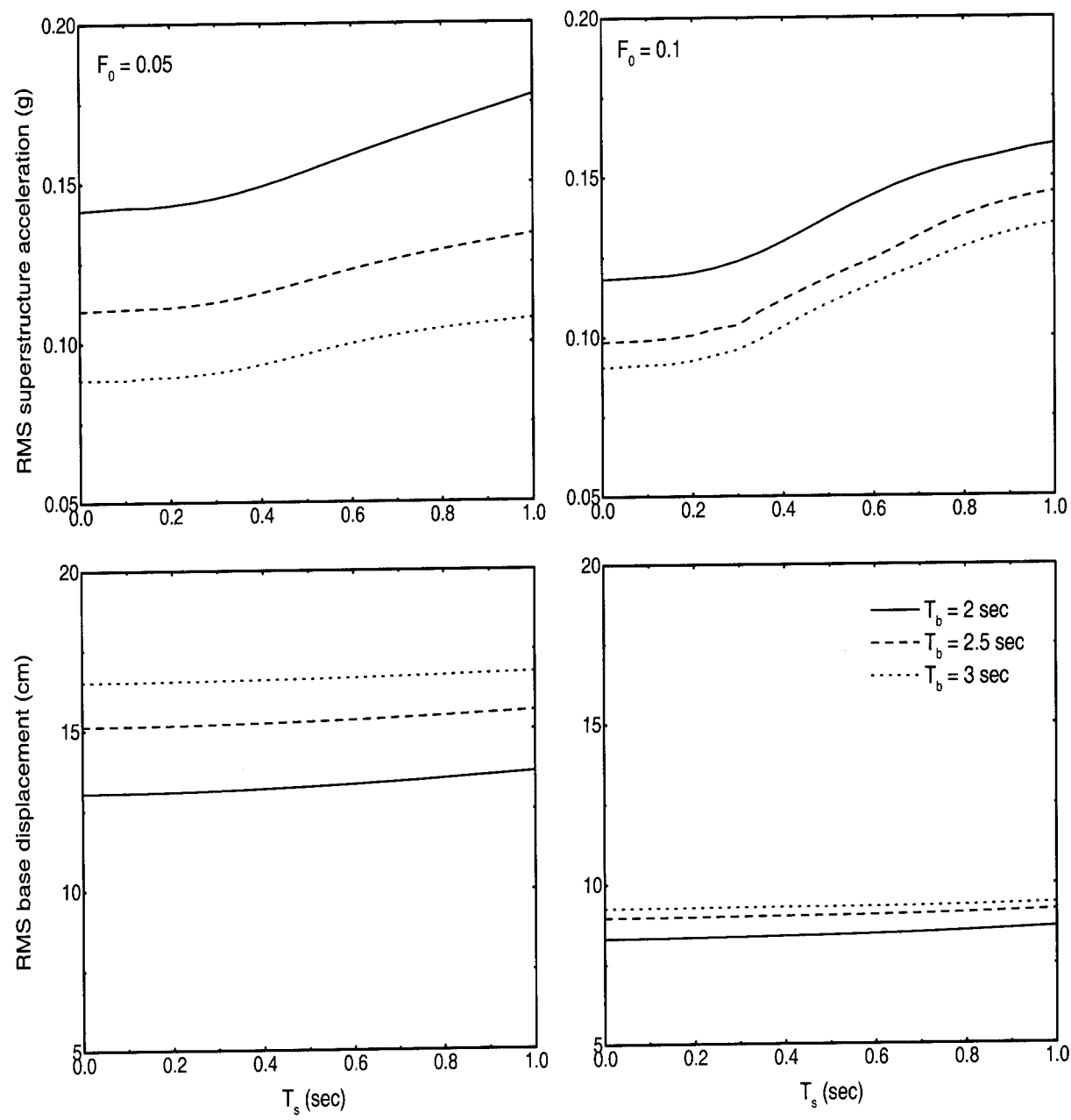

Fig. 8. Effects of the superstructure flexibility on the peak response of structure isolated by the N-Z system $(\xi=0.1)$.

\section{Conclusions}

A comparison of the stochastic response of a baseisolated structure with superstructure modelled as flexible and rigid is made in the present study. The earthquake ground motion is modelled as non-stationary random process specified by the stationary PSDF function and modulating function. The various isolation system considered are the elastomeric bearings and the sliding systems. The effects of the superstructure flexibility on the response of base-isolated structure are investigated under important parametric variation. From the trends of the results of the present study, the following conclusions may be drawn:

1. The stochastic earthquake response of baseisolated structures can be obtained by considering the stationary model of earthquake ground motion with appropriate frequency variation of PSDF function and intensity instead of a nonstationary model.

2. The base displacement in a base-isolated structure obtained by modelling the superstructure as rigid and flexible are found almost identical for all isolation systems. Thus, the rigid superstructure condition can be effectively used for prediction of the bearing displacement in base-isolated structures.

3. The superstructure acceleration in a flexible superstructure obtained by modelling the superstructure as rigid is justified only for very stiff superstructures (i.e. for linear isolation systems the superstructure period should be less that one-fifth of the isolation period).

4. The superstructure acceleration of base-isolated structure will be under-estimated if the flexibility 

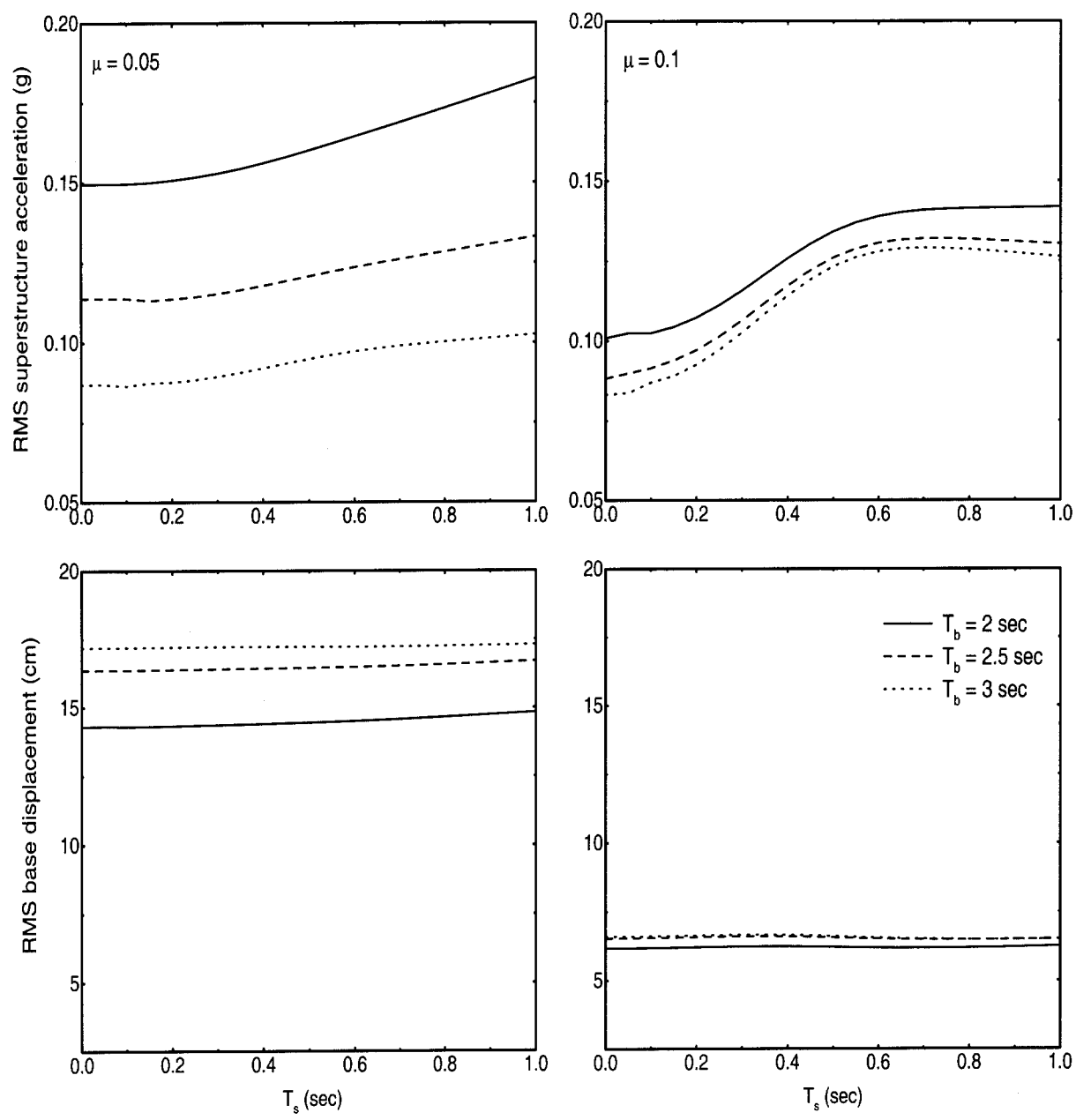

Fig. 9. Effects of the superstructure flexibility on the peak response of structure isolated by the FPS system.

of superstructure is ignored and it is modelled as a rigid body for all types of isolation systems.

5. The difference between the superstructure acceleration obtained by modelling the superstructure as flexible and rigid increases with the increase of the damping, yield strength level and friction coefficient of the isolation system.

6. The difference in the response between flexible and rigid superstructure modelling is not much influenced by the pre-dominant soil frequency.

\section{References}

[1] J.M. Kelly, A seismic base isolation, Review and bibliography, Soil dynamics and Earthquake Engineering 5 (1986), 202216.

[2] I.G. Buckle and R.L. Mayes, Seismic isolation, History, application and performance-A world overview, Earthquake Spec$\operatorname{tra} 6$ (1990), 161-202.
[3] R.S. Jangid and T.K. Datta, Seismic behaviour of base isolated building-A-state-of-the-art-review, Journal of Structures and Buildings 110 (1995), 186-203.

[4] J.C. Simo and J.M. Kelly, The analysis of multi-layer elastomeric bearings, Journal of Applied Mechanics, ASME $\mathbf{5 1}$ (1984), 256-262.

[5] R.I. Skinner, R.G. Tyler, A.J. Hiene and W.H. Robinson, Hysteretic dampers for the protection of structures from earthquakes, Bulletin of New Zealand National Society Earthquake Engineering 13 (1980), 22-36.

[6] N. Mostaghel and J. Tanbakuchi, Response of sliding structure to earthquake support motion, Earthquake Engineering and Structural Dynamics 11 (1983), 729-748.

[7] N. Mostaghel and M. Khodaverdian, Dynamics of resilientfriction base isolator (R-FBI), Earthquake Engineering and Structural Dynamics 15 (1987), 379-390.

[8] R. Gueraud, J.-P. Noel-leroux, M. Livolant and A.P. Michalopoulos, Seismic isolation using sliding elastomer bearing pads, Nuclear Engineering and Design 84 (1985), 363-377.

[9] V.A. Zayas, S.S. Low and S.A. Mahin, A simple pendulum 


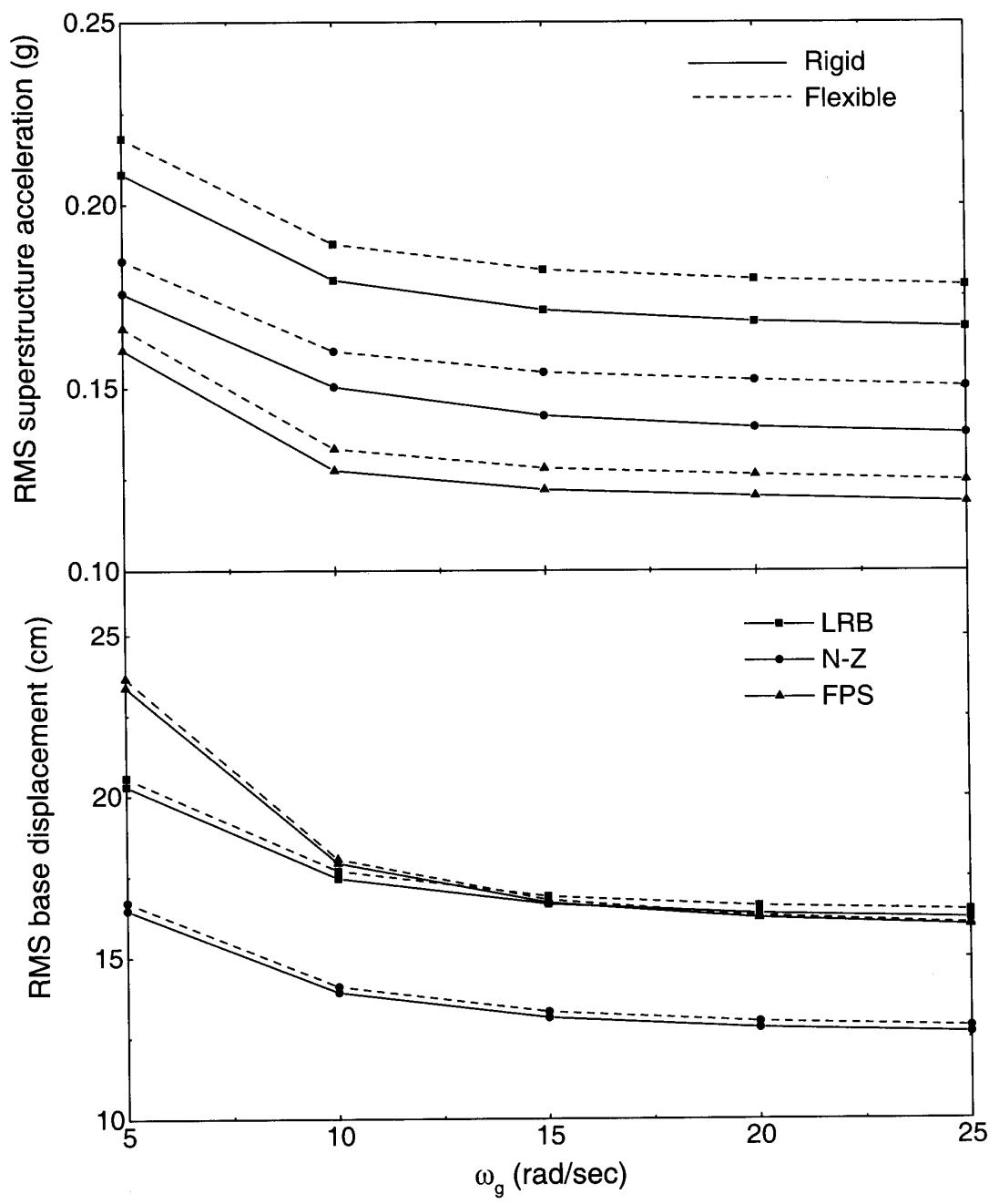

Fig. 10. Effects of soil frequency on the peak response of the base-isolated structure $\left(T_{s}=0.5 \mathrm{sec}\right)$.

technique for achieving seismic isolation, Earthquake Spectra 6 (1990), 317-333.

[10] R.S. Jangid and Y.B. Londhe, Effectiveness of elliptical rolling rods for base-isolation, Journal of Structural Engineering, ASCE 124 (1998), 469-472.

[11] F. Fan and G. Ahmadi, Floor response spectra for base-isolated multi-storey structures, Earthquake Engineering and Structural Dynamics 19 (1990), 377-388.

[12] Y.B. Yang, T.Y. Lee and I.C. Tsai, Response of multi-degreeof-freedom structures with sliding supports, Earthquake Engineering and Structural Dynamics 19 (1990), 739-752.

[13] R.S. Jangid and T.K. Datta, Dissipation of hysteretic energy in base isolated structure, Journal of Shock and Vibration 3 (1996), 353-359.

[14] C.J. Younis and I.G. Tadjbakhsh, Response of sliding rigid structure to base excitation, Journal of Engineering Mechanics, ASCE 110 (1984), 417-432.

[15] Y. Chen and G. Ahmadi, Wind effects on base-isolated structures, Journal of Engineering Mechanics, ASCE 118 (1992), $1708-1727$.
[16] J.M. Kelly, Base isolation, linear theory and design, Earthquake Spectra 6 (1990), 223-243.

117] R.S. Jangid and J.M. Kelly, Base isolation for near-fault motions, Earthquake Engineering and Structural Dynamics 30 (2001), 691-707.

[18] T.C. Liauw, Q.L. Tian and Y.K. Cheung, Structures on sliding base subject to horizontal and vertical motions, Journal of Structural Engineering, ASCE 114 (1998), 2119-2129.

[19] M. Amin and A.H.S. Ang, Non-stationary stochastic model of earthquake motion, Journal of Engineering Mechanics, ASCE 94 (1968), 559-583.

[20] R.W. Clough and J. Penzien, Dynamics of Structures, McGraw Hill Inc., 2nd edition, NY, 1993.

[21] N.C. Nigam, Introduction to Random Vibration, MIT Press, Cambridge, Mass, 1983.

[22] Y.K. Wen, Method of random vibration of hysteretic systems, Journal of Engineering Mechanics, ASCE 102 (1976), 249263.

[23] T.K. Caughey, Equivalent linearization technique, Journal of Acoustics Society of America 35 (1985), 1705-1711. 

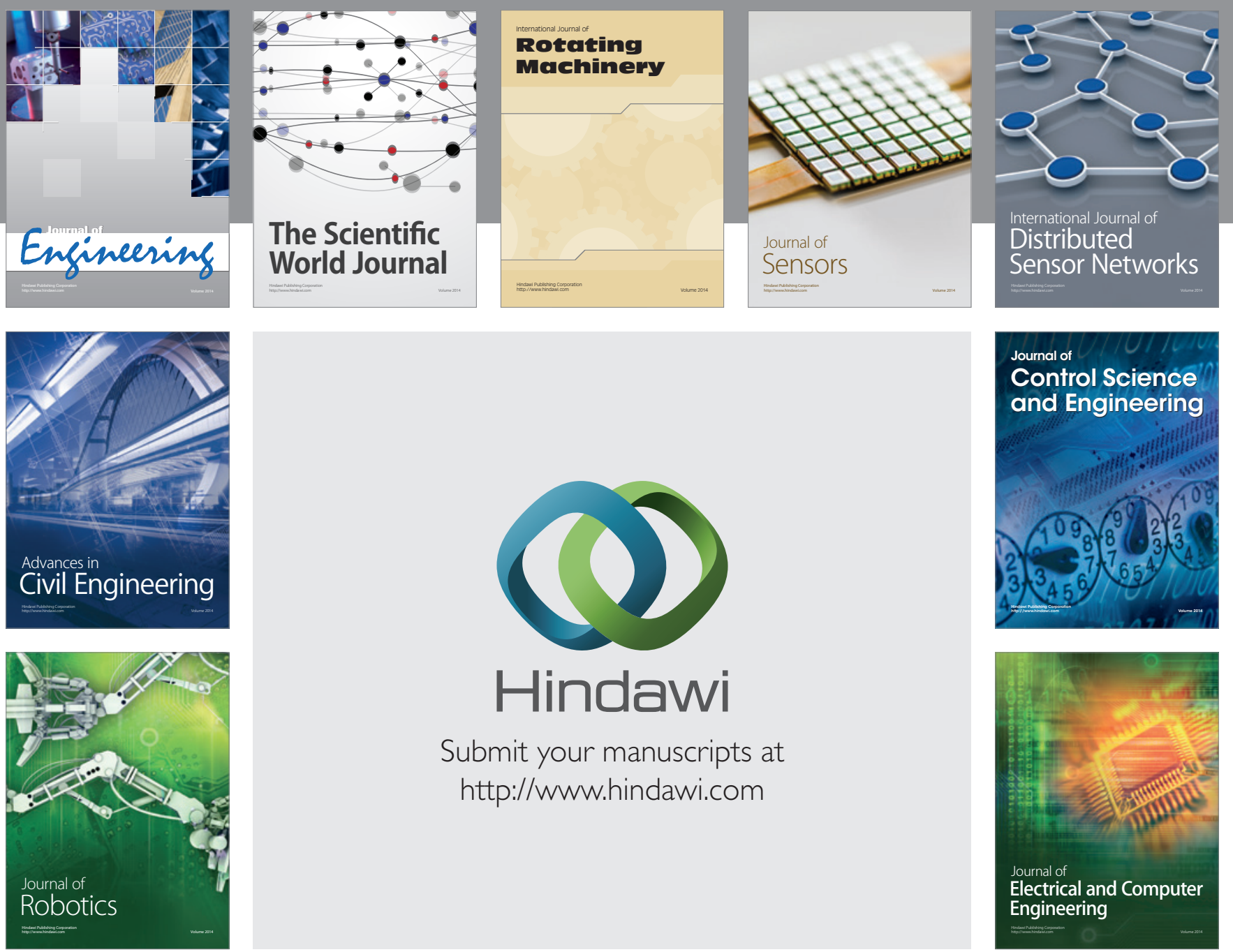

Submit your manuscripts at

http://www.hindawi.com
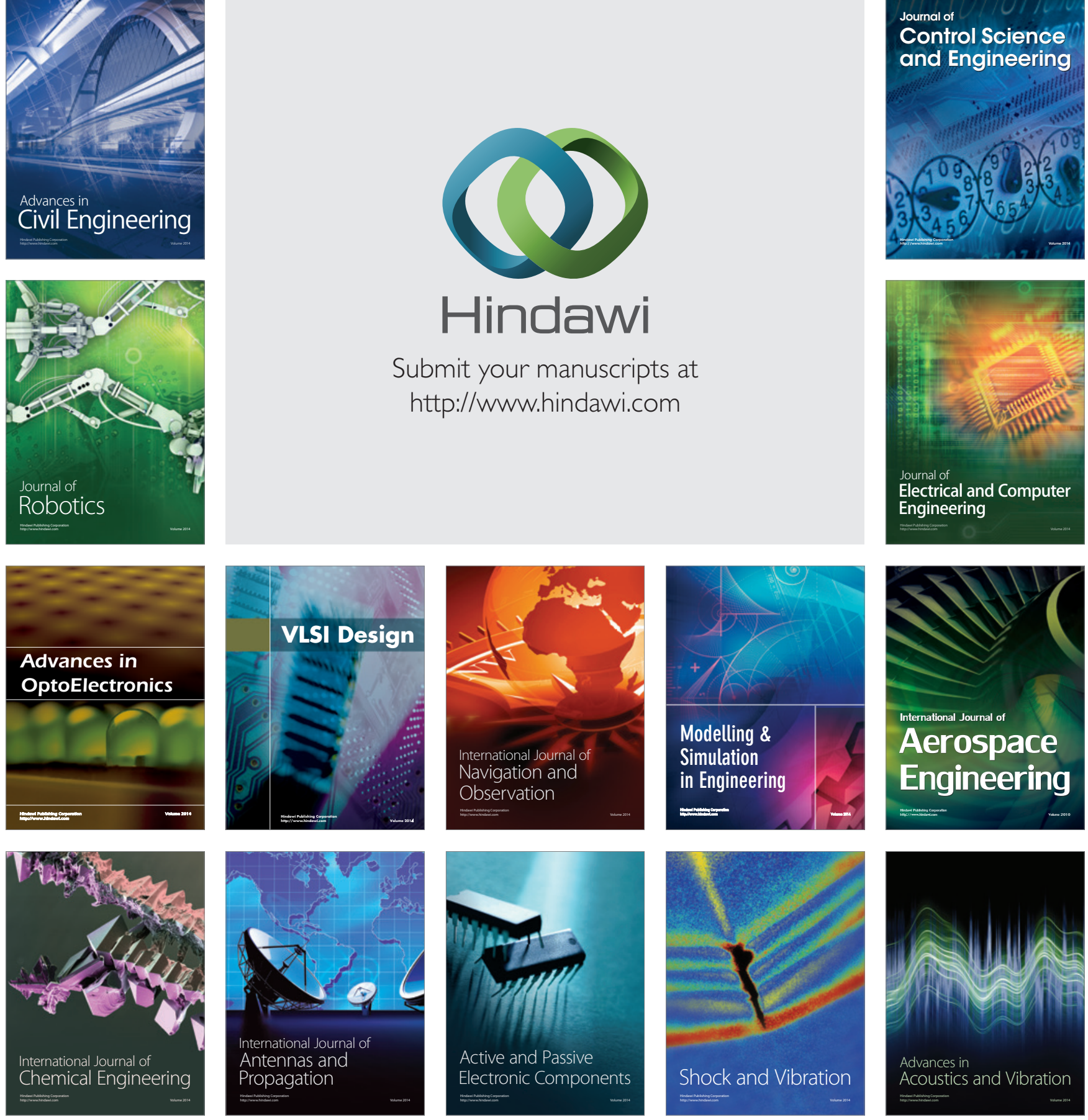